\title{
Research on Marine DAB bidirectional DC converter based on DPS
}

\author{
Chang $\mathrm{Li}^{1}$, Xujing Tang ${ }^{1 *}$, Zhongze Zhang ${ }^{1}$, Yupeng Yuan ${ }^{12}$ \\ ${ }^{1}$ School of Energy and Power Engineering, Wuhan University of Technology, Wuhan, Hubei, 430063, China \\ ${ }^{2}$ National Engineering Research Center for Water Transport Safety, Wuhan, Hubei, 430063, China
}

\begin{abstract}
This paper takes the application of bidirectional DC-DC converter in Marine hybrid power system as the background, the problems of high reflow power and current stress in dual active bridge converter under traditional phase shift control are studied in this paper. Based on the dual phase shift control strategy, the power transmission optimization algorithm model is analyzed and derived, and a control method for optimizing mode switching is proposed. The optimized control algorithm is validated by Matlab/Simulink simulation experiment. The results show that the reflow power and current stress optimization control algorithm can effectively reduce the reflow power and the current stress of the converter, so as to improve the operation performance of DAB converter in high-power ship hybrid power system.
\end{abstract}

\section{Introduction}

Generally, energy storage devices are introduced in the ship microgrid to enhance the dynamic response performance of the ship's power grid. In order to solve the problem of matching the output voltage of the DC bus bar with the energy storage system and ensuring lithium batteries, it is necessary to introduce a two-way between the energy storage device and the DC bus DC-DC converter. However, unlike land-based and vehiclemounted two-way DC-DC, the electric drive system of marine hybrid power needs to respond quickly to large load mutations, which puts forward higher requirements on the dynamic response and power transmission performance of the two-way DC-DC.

The Dual Active Bridge converter has many advantages such as high power density, electrical isolation, fast response speed, and easy implementation of soft switching, and is suitable for applications in medium and high power such as ships [1]. In order to reduce the switching frequency of the switching tube, phase shift control has become the main control method of the current DAB DC converter. Facing the application characteristics of marine high-power transmission, this paper adopts the dual-phase-shift (DPS) control method. In the dual phase shift control, power reflow and current stress are two important factors that affect the performance of the converter[2]. Therefore, the optimal control of power return and current stress has become the focus of current converter research and design. In view of this, this article will introduce the working principle of the converter based on the characteristics of power reflow and current stress under dual phase shift control, derive the mathematical model of the DAB converter, and propose an optimized control scheme for power reflow and current stress based on dual phase shift control[3]. Some references are provided for converter's design.

\section{Working principle}

\subsection{DPS control principle}

The topology of DAB converter is shown in Figure1,which is composed of two H-bridges, inductance $\mathrm{L}$, high-frequency transformer $\mathrm{T}$, capacitance $\mathrm{C}_{f 1}, \mathrm{C}_{f 2}$ etc. $\mathrm{V}_{1}$ and $\mathrm{V}_{2}$ are the voltages on both sides of the converter, the direction of energy flow is controlled by controlling the on-off time of the switch tube. In Figure1, $V_{1}$ and $V_{2}$ are the input or output voltages of the primary and secondary sides. The voltage value and current value of inductance are respectively expressed by $\mathrm{U}_{\mathrm{L}}$ and $i_{\mathrm{L}}$, the ratio of transformer is $\mathrm{n}$, the switching period is represented by $\mathrm{T}_{s}$, For the convenience of analysis, this paper assumes that $\mathrm{V}_{1}$ side is the input $\mathrm{V}_{2}$ side is the output and the voltage variation ratio is $k=V_{1} / n V_{2}$. Dual Phase Shift is to increase the phase shift value inside two full bridges on the basis of single phase shift, and the phase shift angle is the same, so as to reduce the reflow power and improve the working efficiency.

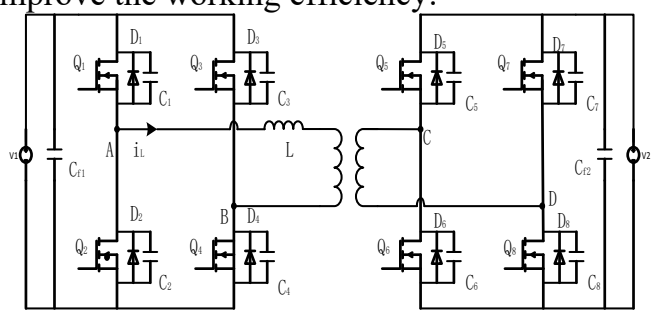

Figure 1. circuit topology of DAB DC converter

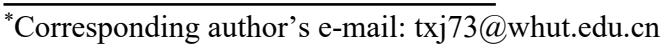


In the DPS control, the inward shift ratio of the dual active bridge converter is $D_{1}$, and the outward shift ratio is $D_{2}$ [4]. The ideal waveform is shown in Figure2.

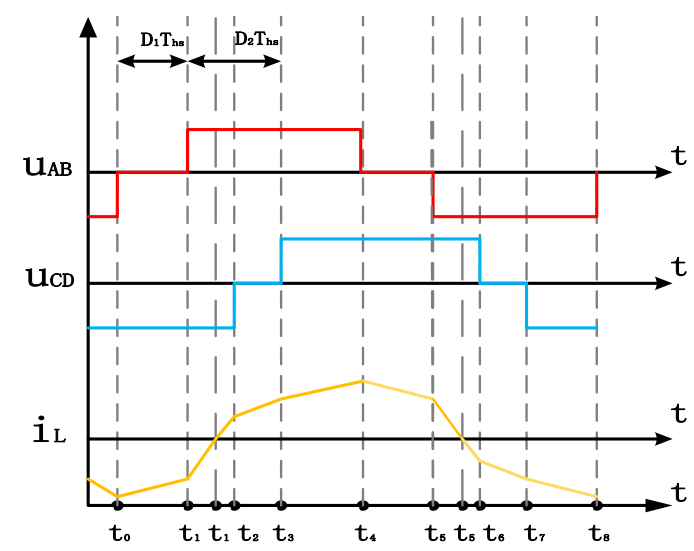

Figure 2. Ideal waveform under dual phase shift control

\subsection{Working mode analysis of DAB converter}

- Working mode $\left.1\left(\mathrm{t}_{0}-\mathrm{t}_{1}\right]\right)$ :

Before entering working mode 1 , the primary side switch $\mathrm{Q}_{2}$ and $\mathrm{Q}_{3}$ are in conduction state, the secondary side diode $D_{6}$ and $D_{7}$ are in conduction state, and $i_{L}$ is negative value. At time $t_{0}$, the original side switch $Q_{3}$ is
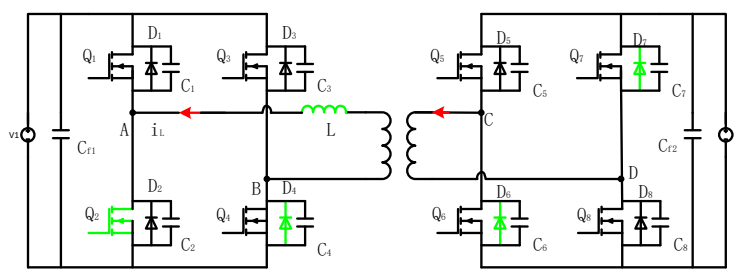

a) working mode1
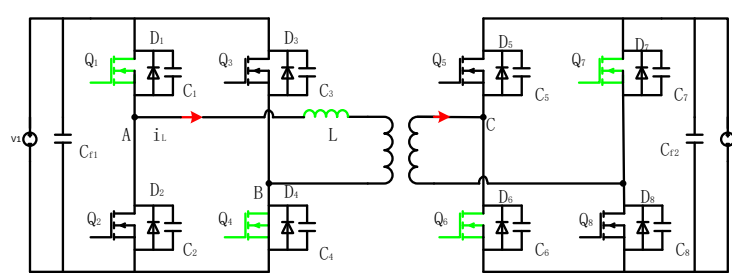

c) working mode3 off, and $D_{4}$ starts to conduct. The on/off state of the secondary side remains unchanged, and the current transmits energy through $D_{6}$ and $D_{7}$. At this point, $u_{L}$ is $n V_{2}$ and the inductive current gradually decreases.

- Working mode $2\left(\mathrm{t}_{1}-\mathrm{t}^{\prime}{ }_{1}\right)$

According to the working principle diagram of DPS, $i_{L}$ is still less than zero at time $t_{1}$. At this time, the original side switch $\mathrm{Q}_{2}$ is off, $D_{1}$ and $D_{4}$ start conduction, and the inductance current continues to flow. The state of the auxiliary side switch tube remains unchanged. The inductance current continues to decrease, and at time $t_{1}^{\prime}$ the inductance current decreases to zero. Under this working mode, the reflow power phenomenon exists on the original edge.

- Working mode $3\left(\mathrm{t}_{1}^{\prime}-\mathrm{t}_{2}\right)$

After time $\mathrm{t}_{1}^{\prime}$, the inductive current $\mathrm{i}_{\mathrm{L}}$ changed from negative to positive. The primary side switch $\mathrm{Q}_{1}$ opens, $\mathrm{Q}_{1}$ and $\mathrm{Q}_{4}$ start conduction, and the secondary side switch $\mathrm{Q}_{6}$ and $\mathrm{Q}_{7}$ start conduction. In this state, power backflow phenomenon exists on the secondary side, making the primary side charge the inductance at the same time. In this case, the inductance voltage $u_{L}$ is $V_{1}+n V_{2}$.

- Working mode $4\left(t_{2}-t_{3}\right)$

At time $t_{2}$ the original side switch $Q_{1}$ and $Q_{4}$ start to conduct; In this mode, the inductance voltage is $V_{1}-n V_{2}$, and the inductance current keeps increasing in a positive way.

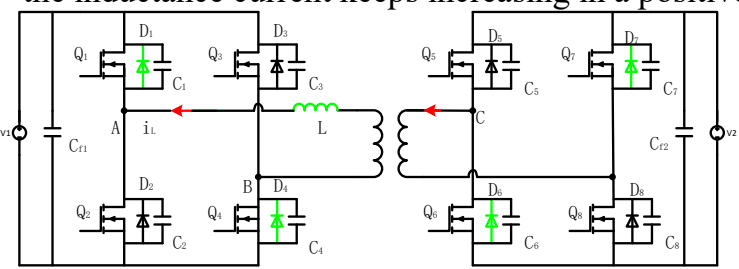

b) working mode2

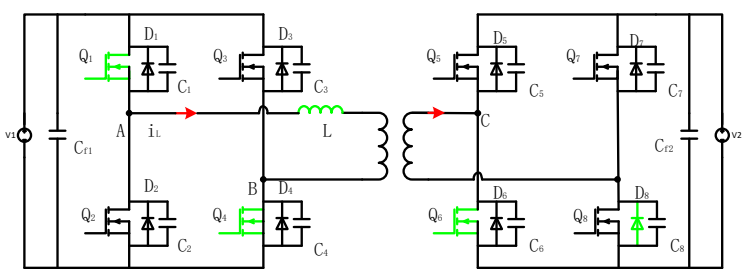

d) working mode4

Figure3. Working mode of DAB under dual phase shift control

\section{Operation Charactertistics}

According to the analysis, it can be known that when $D_{1}+D_{2}>1$, the operating state of the converter has a large fluctuation, and the operating state isn't well[5]. so this paper only studies the operation characteristics analysis of the converter in the case of $D_{1}+D_{2}<1,0<D_{1}<D_{2}<1$.

\subsection{Power characteristic analysis}

In $0<D_{1}<D_{2}<1$ mode, the transmission power model of the dual active bridge converter controlled by DPS can be established by analyzing the working mode and waveform of the dual phase shift:

$$
P_{\mathrm{DPS}}=\frac{1}{T_{h s}} \int_{0}^{T_{h s}} u_{a b} i_{L}(t) d t
$$

In order to facilitate comparative analysis, the maximum transmitted power under single phase shift control is taken as the reference value $P_{N}$, based on which the nominal unit value $p_{D P S}$ can be obtained under double phase shift control.

$$
P_{N}=\frac{n V_{1} V_{2}}{8 f L}
$$




$$
p_{D P S}=\frac{\frac{1}{T_{h s}} \int_{0}^{T_{h s}} u_{a b} i_{L}(t) d t}{\frac{n V_{1} V_{2}}{8 f_{s} L}}=4 D_{2}\left(1-D_{2}\right)-2 D_{1}^{2}
$$

According to formula (3), the transmitted power unit value of DAB converter under DPS control is related to $D_{1}$ and $D_{2}$.

Due to the phase difference between $U_{a b}$ and $U_{c d}$, in the process of power transmission, the original side voltage and the inductive current have opposite phase. As shown in Figure 3, the transmission power of $t_{1} \sim t^{\prime}{ }_{1}$ in this period is opposite to the total average power, which reduces the transmission efficiency of the converter. Therefore, it is called reflow power.

Figure. 2 shows that $i_{L}\left(t_{2}\right)>0$ at time $t_{2}$, so the converter passes zero point $t^{\prime}{ }_{1}$, and the reflow power at stage $\mathrm{t}_{1} \sim \mathrm{t}^{\prime}{ }_{1}$ is:

$$
\mathrm{P}_{\text {cirpPs }}=\frac{\int_{t_{1}}^{t_{1}} u_{a b}|i L(t)| d t}{T_{h s}}=\frac{n V_{1} V_{2}}{16 f L(k+1)}\left[k\left(1-D_{1}\right)+2 D_{2}-D_{1}-1\right]^{2}
$$

Similarly, in order to facilitate the analysis, the reflow power should be normalized to obtain the standard value of reflow power under dual phase shift control:

$$
p_{\text {cirDPS }}=\frac{\left[k\left(1-D_{1}\right)+2 D_{2}-D_{1}-1\right]^{2}}{2(k+1)}
$$

From the above formula, it can be seen that after the input/output voltage ratio is ensured, the reflow power is only related to $\mathrm{D}_{1}, \mathrm{D}_{2}$.

\subsection{Current stress analysis}

Current stress is the maximum current value of DAB converter circuit during operation. Excessive current stress not only causes excessive loss of the converter, but also increases the requirement of the device and increases the production cost[6]. Therefore, current stress analysis is an important procedure for converter design.

Combined with Figure 2, it can be seen that when $k \geq 1$, the maximum value of inductance current stress of $\mathrm{DAB}$ converter under the control of dual phase shift is located at time $\mathrm{t}_{0}$.

$$
i L_{\max }=i L\left(t_{0}\right)=\frac{n V_{2}}{4 f L}\left[\left(D_{1}-1\right) k+1-D_{1}-2 D_{2}\right]
$$

According to Equations (6) and (1), the nominal value of the inductance current stress $\mathrm{G}_{\mathrm{Lmax}}$ can be obtained.

$$
\mathrm{G}_{\mathrm{L} \max }=\frac{i_{L \max }}{i_{N}}=2\left(k-1+2 D_{2}+D_{1}-k D_{1}\right)
$$

\section{Design of Phase Shift Control System}

Combined with the characteristics of the ship's flexible operation state, this paper designs a phase shift control system of the Marine DAB DC converter that can be used for mode switching based on the optimization control algorithm.

\subsection{Design of phase shift control algorithm}

\subsubsection{Control based on minimum reflow power}

Under the condition of transmission power $p$ and voltage ratio $\mathrm{k}$ are setted, the Lagrange function is established with the minimum reflow power as the optimization objective, and the control method of the minimum reflow power under the control of double phase shift is analyzed and deduced.

$$
\begin{aligned}
& \text { Minimize } p_{\text {cir } D P S}=\frac{\left[k\left(1-D_{1}\right)+2 D_{2}-D_{1}-1\right]^{2}}{2(k+1)} \\
& \text { Subject to }\left\{\begin{array}{l}
2\left(-D_{1}^{2}+2 D_{2}-2 D_{2}^{2}\right)-p_{0}=0 \\
1>D_{1}>0,1>D_{2}>0 \\
D_{1}-D_{2}<0
\end{array}\right. \\
& L\left(D_{1}, D_{2}, \lambda\right)=\frac{\left[k\left(1-D_{1}\right)+2 D_{2}-D_{1}-1\right]^{2}}{2(k+1)}+\lambda\left(2\left(-D_{1}^{2}+2 \mathrm{D}_{2}-2 \mathrm{D}_{2}{ }^{2}\right)-p_{0}\right)
\end{aligned}
$$

According to the derivation of Lagrangian function, the mathematical relationship between the externally shifted phase called $D_{1}, D_{2}$ and the voltage change ratio $\mathrm{k}$ can be obtained.

$$
\left\{\begin{array}{l}
\frac{\partial L}{\partial D_{1}}=0 \\
\frac{\partial L}{\partial D_{2}}=0
\end{array}\right.
$$

According to Equation (8), (10) and (11), it can be obtained:

$$
D_{2}=\frac{k\left(1+D_{1}\right)-2 D_{1}-1}{2(k-1)}\left(\frac{2 k-2}{k^{2}} \leq p \leq 1\right)
$$

According to (10) and (12), the combined solution of the optimal phase shift Angle under the variable voltage ratio $\mathrm{k}$ and power transmission $\mathrm{p}$ can be obtained respectively:

$$
\left\{\begin{array}{l}
D_{1}=(k-1) \sqrt{\frac{1-p}{(k-1)^{2}+1}} \\
D_{2}=\frac{1}{2}+\frac{k-2}{2} \sqrt{\frac{1-p}{(k-1)^{2}+1}}\left(\frac{2 k-2}{k^{2}} \leq p \leq 1\right)
\end{array}\right.
$$

\subsubsection{Optimal control based on current stress}

In order to obtain the optimal phase shift ratio combination solution under the minimum current stress, the method of constructing Lagrange function is adopted in this paper[7].In the mode of $0<D_{1}<D_{2}<1$, the perunit value of the inductance electric stress at time $t_{0}$ is taken as the objective function. In order to obtain the minimum value of the objective function, the expression under multi-constraint conditions is obtained as follows:

Minimize $\mathrm{G}_{\mathrm{L} \max }=\frac{i_{L \max }}{i_{N}}=2\left(k-1+2 D_{2}+D_{1}-k D_{1}\right)$ 


$$
\text { Subject to }\left\{\begin{array}{l}
2\left(-\mathrm{D}_{1}{ }^{2}+2 \mathrm{D}_{2}-2 \mathrm{D}_{2}{ }^{2}\right)-p_{0}=0 \\
1>D_{1}>0,1>D_{2}>0, \\
D_{1}-D_{2}<0
\end{array}\right.
$$

Construct Lagrange function:

$\mathrm{L}\left(\mathrm{D}_{1}, \mathrm{D}_{2}, \lambda\right)=2\left(\mathrm{k}-1+2 \mathrm{D}_{2}+\mathrm{D}_{1}-\mathrm{k} \mathrm{D}_{1}\right)+\left[2\left(-\mathrm{D}_{1}^{2}+2 \mathrm{D}_{2}-2 \mathrm{D}_{2}^{2}\right)-p_{0}\right]$

Solving the above equation, the optimal solution can be obtained as follows:

$$
\left\{\begin{array}{l}
D_{1}=(k-1) \sqrt{\frac{1-p}{2 k^{2}+6-4 k}} \\
D_{2}=-\sqrt{\frac{1-p}{2 k^{2}+6-4 k}}+\frac{1}{2}
\end{array}\right.
$$

By substituting the above formula into the peak current, the peak current value can be solved:

$$
G_{L \text { max }}=2 k-\sqrt{(1-p)\left(2 k^{2}+6-4 \mathrm{k}\right)}
$$

\subsection{Design of phase shift control system}

According to the working principle of the converter phase shift control, the minimum reflow power control and current stress control are based on the dual phase shift control. Firstly, the voltage and current signals on both sides of high and low voltage are collected through the acquisition module. The input-output converter ratio $\mathrm{k}$ and the power required by the low-pressure side ship load $\mathrm{p}_{0}$ can be calculated; Then, according to the formula, it can be calculated the Internal and external shift ratio $D_{1}$ and $\mathrm{D}_{2}$ and control the pulse signal generated by the pulse modulator to drive the switching tube's opening and closing time.

Based on the above analysis, this paper proposes a dual phase shift control scheme for optimal control of reflow power and switching of current stress modes. As is shown in Figure 4.

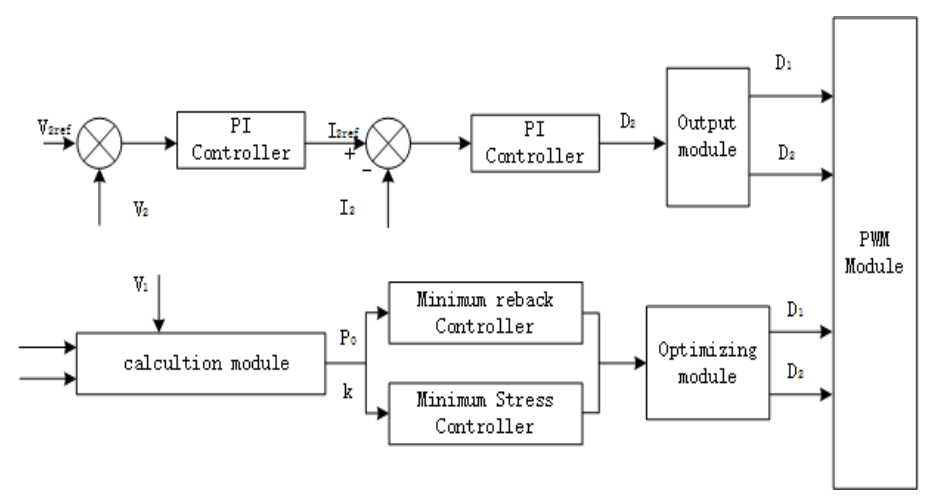

Figure 4. Optimized control system of DAB

\section{Simulation Analysis}

In order to verify the effectiveness of the control scheme under dual phase shift control, the simulation model of dual active bridge converter is built in the Matlab Simulink simulation platform. By comparing the waveform effects of traditional single phase shift control and double phase shift control, the effectiveness of the proposed double active bridge converter double phase shift control scheme is verified.
Figure 5 and 6 respectively show the inductance current waveforms based on traditional phase shift control and double phase shift control under given working conditions. According to the figure, under the same transmission power, the operating current stress of the proposed optimal control scheme is lower than that of the traditional control scheme, which can reduce the switching loss and improve the operating safety performance of the converter.

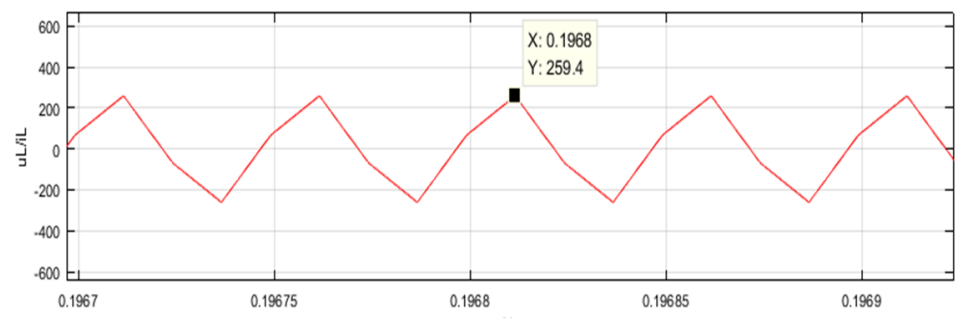

Figure 5. Inductance current curve under SPS control 


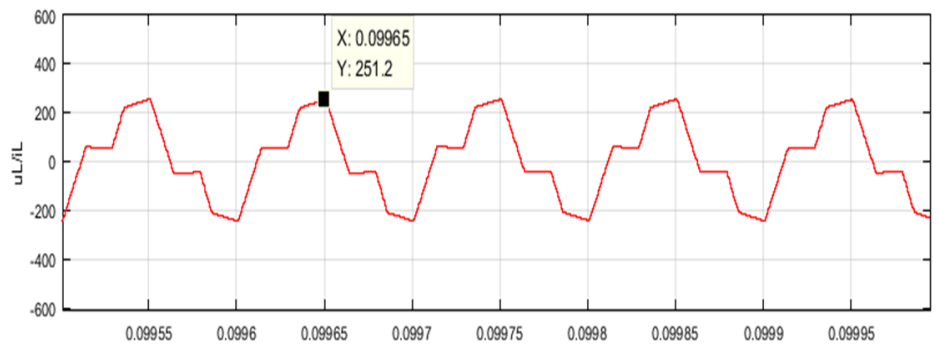

Figure 6. Current stress curve under DPS control

Figure 7 and 8 respectively show the voltage and inductance current waveform of the primary side of the converter under a given working condition. Through comparison, it can be seen that under the same transmission power, the reflow power of the primary side can be reduced by the optimal control scheme of reflow power based on double phase shift. Therefore, when the transmission power is constant, the optimal control scheme can suppress the generation of reflow power and improve the efficiency of the converter.

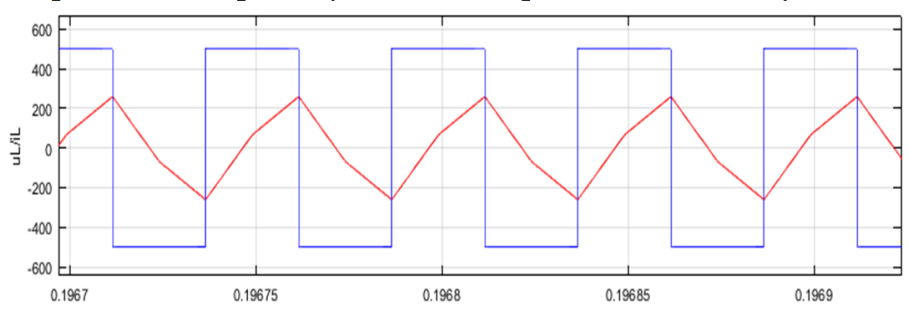

Figure 7.Reflow power curve under SPS control

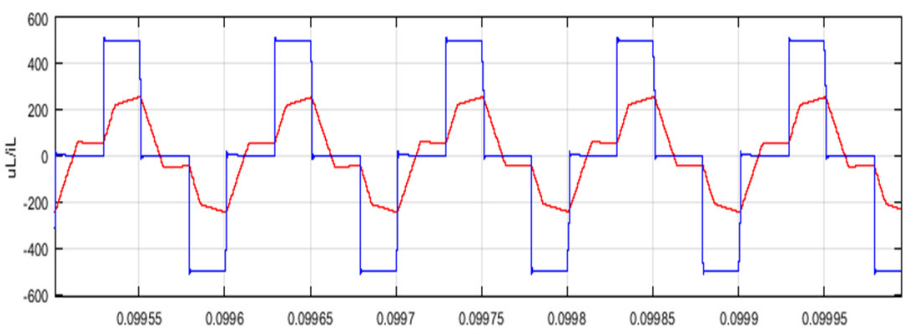

Figure 8. Reflow power curve under DPS control

\section{Conclusion}

Through the comparative analysis of the experimental results, it can be seen that the reflow power control scheme proposed in this paper can effectively reduce the relow power of the converter, and the current stress optimization control can reduce the current stress of the converter. It ensures the safety and stability of the converter in the start-up and operation of the high-power ship energy storage system, improves the working efficiency of the converter, and provides a reference for the design of the high-power DAB converter for ships.

\section{Acknowledgments}

This paper is sponsored by High-tech Ship Scientific Research Project [2018] NO.473 of The Ministry of Industry and Information Technology of China.

\section{References}

1. Sun K, Chen H, Wu H.F A review of anlysis method and control technology for isolated bidirectional DCDC converter used in energy storage systems [J] Advanced Technology of Electrical Engineering and Energy 2019,38(8):1-9

2. Liu b,Shen A.D, Gao D.J Research and design of bidirectional DC-DC converter for hybrid power ships [J] Modern Electronic Technology.2014.12(6),127131

3. Wang, Y, Wang, F, Lin, Y, Hao, T. (2018). Sensorless parameter estimation and current-sharing strategy in two-phase and multiphase IPOP DAB DC-DC converters. IEEE Power Electronics, 11(6), 11351142.

4. Sha guang-lin, Research on double Active Bridge DC-DC Converter in Power electronic Transformer [D].2016. Wuhan

5. Wang Y.B, Wang S, Feng B, Optimal Current Control Strategy of Dual-Active-Bridge DC-DC 
Converter Based on Dual-Phase-Shift Control [J] Transaction of China electro technical society,

6. Lu, J., Wang, Y., Li, X., \& Du, C. (2018). HighConversion-Ratio Isolated Bidirectional DC-DC Converter for Distributed Energy Storage Systems. IEEE Transactions on Power Electronics, 1-1.

7. Liu, P., Chen, C., Duan, S., \& Zhu, W. (2017). Dual Phase-Shifted Modulation Strategy for the ThreeLevel Dual Active Bridge DC-DC Converter. IEEE Transactions on Industrial Electronics, 64(10), 78197830 . 